handensein einiger Parteien mache bereits eine Demokratie aus. Angesichts der nur begrenzten Meinungsfreiheit auf den Philippinen sollte man aber Verständnis für manche Unterlassungen haben.

Klaus Kähler

\title{
Mexican Labor Law
}

Commerce Clearing House Inc., Chicago/Ill. 1974, 489 S.

Mit dieser spanisch-englischen Textausgabe wird das am 1. Mai 1970 in Kraft getretene neue mexikanische Bundesarbeitsgesetz (Ley Federal del Trabajo) in der Fassung vom 10. Januar 1974 vorgelegt. Im liberalen Staat des 19. Jahrhunderts fand der Arbeitnehmer in der Gesetzgebung keine besondere Berücksichtigung. Zwar ließ die allgemeine Vereinigungsfreiheit in der Verfassung von 1857 Hilfsvereinigungen auf Gegenseitigkeit unter Arbeitnehmern zu, aber die Wirklichkeit unter der Herrschaft Diáz wurde geprägt etwa durch das Strafgesetzbuch des Bundes-Distrikts, das jedwede einseitige Durchsetzung von Lohnerhöhungen oder -herabsetzungen unter Strafe stellte. Erst die Verfassung von 1917, diese dann allerdings als erste Verfassung überhaupt, verbürgte in Art. 123 Rechte der Arbeitnehmer neben den Rechten aus Art. 4 - Recht auf die Ausübung eines jeden erlaubten Berufs, Gewerbes, Handels oder Arbeit - und Art. 5 - Arbeit nur gegen angemessene Vergütung und aufgrund freien Willensentschlusses - 1929 erhielt der Bundesstaat kraft Verfassungsänderung - Art. 73 Abs. X - die ausschließliche Gesetzgebungskompetenz für das Arbeitsrecht, von der er mit dem Bundesarbeitsgesetz vom 18. August 1931 Gebrauch machte. Dieses Gesetz wurde in der Folgezeit mehrfach geändert, vor allem aus Anlaß der Übernahme von Konventionen der Internationalen Arbeitsorganisation. Hauptsächlichste Rechtsquelle des geltenden Arbeitsrechts ist das Bundesarbeitsgesetz 1970 mit seinen 16 Titeln und 12 Übergangsbestimmungen. Nach den allgemeinen Grundsätzen in Art. $1-19$, so z. B. das Gebot arbeitnehmerfreundlicher Auslegung der Normen, Art. 18 Satz 2, enthaltend, wird im II. Titel der Individualarbeitsvertrag geregelt: Der Arbeitsvertrag, definiert in Art. 20, muß schriftlich und grundsätzlich auf unbestimmte Zeit abgeschlossen sein, Art. 24, 25 und 35. Das mexikanische Arbeitsrecht unterscheidet nicht zwischen Arbeitern und Angestellten und kennt keine Sonderrechte für Lehrlinge, die daher von Anfang an den vollen Rechten und Pflichten eines Arbeitnehmers unterliegen. Sonderregeln, Art. $182 \mathrm{ff}$., gelten allerdings für die „trabajadores de confianza“, Art. 9 Abs. II, denen es ihrer Nähe zum Arbeitgeber wegen, anders also als nach der deutschen Gewerkschaftspolitik, nicht erlaubt ist, den allgemeinen Syndikaten anzugehören, Art. 183.

Kündigungsfristen kennt das Gesetz nicht. Vielmehr können Arbeitnehmer und Arbeitgeber jederzeit das Arbeitsverhältnis beendigen, wenn ihnen ein rechtfertigender Grund zur Seite steht (aufgezählt in Art. 47 einerseits und Art. 51 andererseits). Das kollektive Arbeitsrecht wird in den Titeln VII - X geregelt: Die Art. $354 \mathrm{ff}$. spezifizieren die bereits verfassungsrechtlich garantierte Koalitionsfreiheit, insbesondere auch die hier ausdrücklich erwähnte negative Koalitionsfreiheit, Art. 358. Allerdings läßt Art. 395 die Einführung der „clausula de exclusión de ingreso" durch Kollektivvertrag zu, so daß für alle Neueinstellungen die Zugehörigkeit zur vertragschließenden Gewerkschaft gefordert werden kann'. Über-

1 Schulenburg, Das kollektive Arbeitsrecht in Mexico, Stuttgart 1973, S. 25 (bespr. in VRU 1975, S. 299). 
haupt hat ein Arbeitgeber bei Neueinstellungen Gewerkschaftsangehörige freien Arbeitnehmern bei ansonsten gleichen Voraussetzungen vorzuziehen, Art. 154. Auf diese Bestimmung wird auch in Art. 438 verwiesen im Fall der Wiedereröffnung des Betriebes durch den Arbeitgeber. Für nichtmexikanische Arbeitskräfte ist auf Art. 7 hinzuweisen, wonach in jedem Unternehmen wenigstens 90 Prozent der Arbeitnehmer Mexikaner sein müssen mit Ausnahme der Direktionsetage (Abs. II ebd.).

Gerhard Scheffler

ERnst U. Petersmann

Wirtschaftsintegrationsrecht und Investitionsgesetzgebung der Entwicklungsländer - Grundprobleme, rechtsvergleichende und multidisziplinäre Aspekte

Nomos Verlagsgesellschaft, Baden-Baden 1974, 253 S.

Mit dieser Untersuchung über Rechtsaspekte der Wirtschaftsintegration und der Behandlung ausländischer Investitionen in Entwicklungsländern sowie über die rechtstatsächliche Integrations- und Investitionspraxis stellt die Nomos Verlagsge. sellschaft ihre neue Schriftenreihe zur Entwicklungspolitik vor. Die Arbeit, entstanden am Max-Planck-Institut für ausländisches öffentliches Recht und Völkerrecht in Heidelberg, ist aus einer umfassenderen Untersuchung des Autors über Entstehung und Dynamik des „Entwicklungsvölkerrechts“ hervorgegangen. Der Autor gibt an, daß er in einer „... für völkerrechtliche Monographien noch immer unüblich ,multidisziplinären" Weise" (S. 13) gearbeitet hat. Multidisziplinär bedeutet in diesem Zusammenhang, daß die politisch-wirtschaftlichen Aspekte der analysierten Problematik nicht völlig ausgeklammert werden.

Einleitend untersucht Petersmann das wirtschaftliche Integrationsrecht als Teil eines in der Entstehung begriffenen „Entwicklungsvölkerrechts“. Nachdem er in Kapitel 1 die grundlegenden wirtschaftlichen Probleme der Integration aufgezeigt hat, prüft er in Kapitel 2 die Aufgaben und Perspektiven einer völkerrechtlichen Integrationstheorie. Integration ist ein Prozeß, der wirtschaftliche, politische, kulturelle und rechtliche Aspekte des gesellschaftlichen Systems umfaßt, die in einem sich ergänzenden und teilweise bedingenden Beziehungszusammenhang stehen. Die im Integrationsprozeß in den einzelnen Teilbereichen auftretenden Probleme sind entsprechend dem Entwicklungsniveau, den historischen und sonstigen regionalen Besonderheiten des "Integrationsgebildes" und seiner Mitglieder unterschiedlich. Im Einklang damit formuliert Petersmann als unmittelbare Aufgabe und gleichzeitig Voraussetzung für die mögliche Erarbeitung einer völkerrechtlichen Integrationstheorie überhaupt „... die Systematisierung und Erklärung der rechtlichen Parallelen und Unterschiede im jeweiligen regionalen Integrationsrecht ... sowie eine auf dem empirischen Vergleich von Integrationszielen und Integrationserfolgen beruhende Bewertung der jeweiligen Formen und Methoden des Integrationsrechts.“ (S. 51) In den anschließenden Kapiteln wird eine übersichtsartige Darstellung der bestehenden Integrationsabkommen in Lateinamerika, Asien, Afrika und im Nahen Osten sowie eine Erörterung ihrer institutionellen Aspekte vorgenommen. Völkerrechtsaspekte der subregionalen Integrationsbanken als Instrumente der Kapitallenkung und des Finanzausgleichs ergänzen diesen Teil.

Einen etwa gleich umfangreichen Teil widmet dann der Autor den rechtlichen Fragen von Investitionsförderung, industrieller Integration und ausländischen Direkt- 